\title{
Improving the angular resolution of coded aperture instruments using a modified Lucy-Richardson algorithm for deconvolution.
}

\author{
L. Sambo, ${ }^{a}$ J.B. Stephen, ${ }^{*}$ S. Bonettini, ${ }^{c}$ G.Zanghirati, ${ }^{c d}$ and F.Frontera ${ }^{a}$ \\ ${ }^{a}$ Department of Physics, University of Ferrara, via Saragat 1, 44100 Ferrara, Italy \\ ${ }^{b}$ INAF/IASF-Bologna, Via P. Gobetti 101, 40129 Bologna, Italy \\ ${ }^{c}$ Department of Mathematics, University of Ferrara, via Saragat 1, 44100 Ferrara, Italy \\ d "Math4Tech" Center, University of Ferrara, via Saragat 1, 44100 Ferrara, Italy \\ E-mail: sambodfe.infn.it, stephen@iasfbo.inaf.it, \\ silvia.bonettini@unife.it, g.zanghirati@unife.it, \\ fronteraefe.infn.it
}

\begin{abstract}
A problem with coded-mask telescopes is the achievable angular resolution. For example, with the standard cross-correlation (CC) analysis, the INTEGRAL IBIS/ISGRI angular resolution is about 13'. We are currently investigating an iterative Lucy-Richardson (LR) algorithm. The LR algorithm can be used effectively when the PSF is known, but little or no information is available for the noise. This algorithm maximizes the probability of the restored image, under the assumption that the noise is Poisson distributed, which is appropriate for photon noise in the data, and converges to the maximum likelihood solution. We have modified the classical LR algorithm, adding non-negative constraints. It doesn't take into account of the features leading to a difference in PSF depending on position in the field of view (dead pixels, gaps between modules etc), which are easily corrected for in the classical CC analysis, so we must correct for these either after the restoration of the image or by modifing the data before the sky reconstruction. We present some results using real IBIS data indicating the power of the proposed reconstruction algorithm.
\end{abstract}

The Extreme sky: Sampling the Universe above $10 \mathrm{keV}$ - extremesky2009,

October 13-17, 2009

Otranto (Lecce) Italy

\footnotetext{
* Speaker.
} 


\section{Introduction}

The process of image formation can be described with the system of linear equations $P f+n=$ $d$ where $P$ is the PSF, $f$ is the unknown object, $d$ are data and $n$ is noise.

This is true also for coded mask telescopes as IBIS/Integral (8), where the images are usually formed using the CC techique (2), where the intensity is given by $c=P^{T} d$, given by multiplying Eq. 1 by the transpose of $P$. Some of the disadvantages of the $\mathrm{CC}$ decoding is the presence of negative values in $c$, and of 'ghost' sources which must be cleaned from the final image.

At the beginning of the 90's the Chinese scientists Li T.P. e Wu. M. (5, 3) proposed to directly solve the image equation using Direct Demodulation. To make the deconvolution from the data to the object space, they wrote a bounded minimum problem that is solved with iterative algorithms including LR.

We have started an investigation into the possible advantages of using a LR-type iterative deconvolution technique in order to overcome some of the limitations in the $\mathrm{CC}$ reconstruction as mentioned above. In this preliminary analysis presented herein, we have used both simulated and real data from the IBIS/ISGRI instrument (4), but without taking into account complications such as energy band selection, instrumental features, instrumental noise, etc.

\section{The Lucy-Richardson algorithm}

The LR algorithm $(6,7)$ maximizes the probability of the restored image, under the assumption that the noise is Poisson distributed, which is appropriate for photon noise in the data, and converges to the maximum likelihood solution.

It's an iterative technique where the $\mathrm{k}^{\text {th }}$ estimate of the decoded sky is derived from the $(\mathrm{k}-1)^{t h}$ estimate by:

$$
x^{k}=x^{k-1} M^{T} \frac{d}{M x^{k}+b}
$$

where $M$ is the PSF of the instrument and $b$ is the background. The non-negativity is guaranteed by the Eq.2.1 itself.(1)

Choices that affect the quality of the reconstructed image are:

- The iteration number $k$ plays the role of regularization parameter: it should be chosen so that there is the minimum amount of noise amplification.

- The system PSF must be constructed in the most realistic way, to include all features that modify the original signal.

- The initial image must be chosen carefully. Pixels with zero counts in the initial image remain zero in the reconstructed image. If the initial image is non-negative, all the following iterations have non-negative values.

\section{Check in terms of spatial resolution and sensitivity}

We have simulated data obtained using the ISGRI instrument of INTEGRAL/IBIS. A typical data distribution consists of a $128 \times 128$ array of photon counts over a $130 \times 134$ geometrical matrix 
(due to the gaps between element modules). The data are then introduced into the iterative analysis using the mask pattern (resampled at the same level as the data) as the PSF.

\subsection{Results with simulated data}

We have simulated two strong sources in the FCFOV at varying angular separations, adding a Poisson background distribution with constant mean value of 5000 counts/pxl and fixed the iteration number to the constant value of 200 .

Fig. 1 shows a situation where the two equal intensity sources are separated by the theoretical resolution limit of 13 arcminutes. The image is deconvolved using a standard correlation analysis (left) where, while it is evident that the two sources are not consistent with the PSF, they are not resolved. On the right it's showed the LR deconvolution output where the two sources are clearly well separated. In Fig.1 are also shown the line profiles of the reconstructed source.

We also simulate one source with a varying intensity with respect to the background (Fig.2).
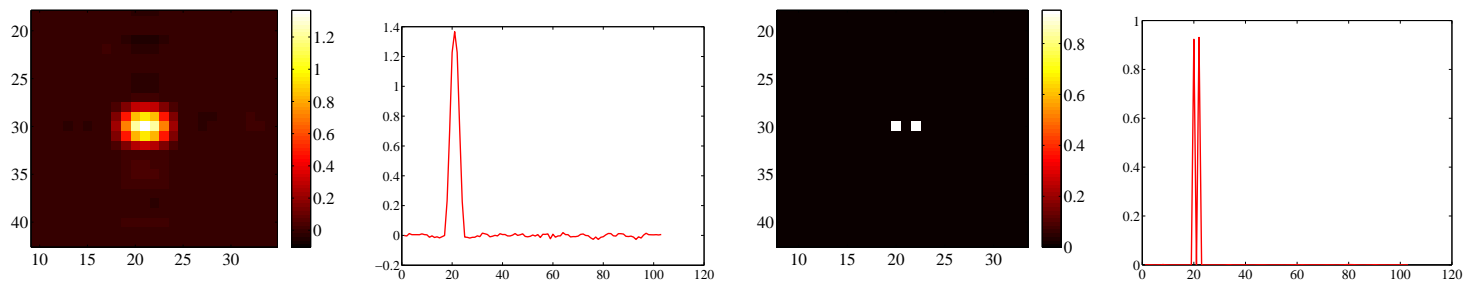

Figure 1: Spatial resolution analysis: reconstructions and line-profiles (vertical one) of the FCFOV with CC (left) and LR algorithm (right).

The situation where the source is scaled by a factor of 0.01 is shown. The scale factor affects the SNR and the value of the intensity peak.
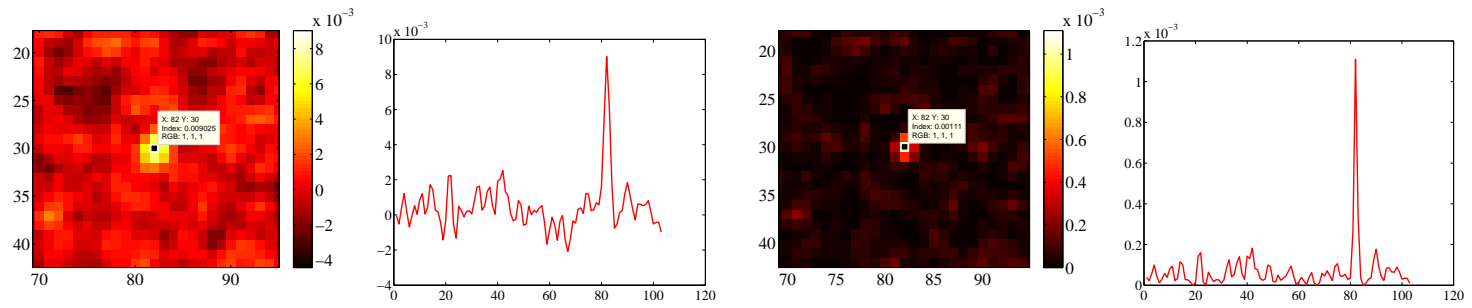

Figure 2: Minimum intensity detection: reconstructions and profiles (vertical one) of the FCFOV with CC (left) and LR algorithm (right).

\subsection{Results with IBIS/Integral data}

We have started using also real Science Windows from INTEGRAL/IBIS in order to perform a preliminary investigation of the LR applicability to real data. We have used four SCW (a GRB, one around the Crab, one around Cygnus X-1 and another in the Galactic Centre). Fig.3 show the results of the analysis using standard correlation analysis (up) and the LR algorithm (down).

LR doesn't into account of the features leading to a difference in PSF depending on position in the field of view (dead pixels, gaps between modules etc), which are easily corrected for in the classical 

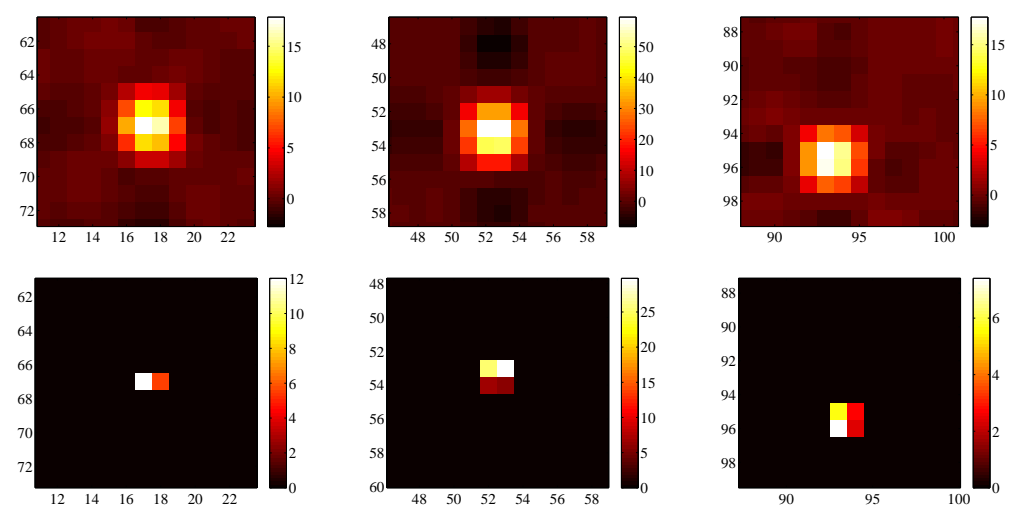

Figure 3: Real data analysis: FCFOV reconstruction with standard CC software (up) and LR algorithm(down). In the order, there are results of a GRB, around the Crab and around Cygnus X-1.

$\mathrm{CC}$ analysis. We must correct for these either after the restoration of the image or by modifing the data before the sky reconstruction.

\subsection{Study of the Galactic Center science window.}

We present a detailed study of the SCW in the Galactic Centre with pointing coordinates $(\mathrm{ra}, \mathrm{dec})=(174617.18 ;-2859$ 58.8). In Tab. 1 are reported data of the four main sources that we can resolve with LR algorithm. As we can see in the upper Fig.4, LR algorithm reconstrucs sources with an higher SNR and with a better resolution than CC.

Until now we have imposed that all sources have an equal probability to be in the FCFOV or in the

\begin{tabular}{|c|c|c|c|c|c|}
\hline Pixel & FCX & FCY & CorrInt & LRInt & OSA Corrispondence \\
\hline A & 101 & 12 & 4.73 & 1.96 & GX 5-1 \\
B & 93 & 12 & 5.04 & 1.27 & GRS 1758-258 \\
C & 79 & 17 & 2.01 & 0.43 & 1E 1740.7-2942 \\
D & 2 & 50 & 1.72 & 0.26 & GX 354-0 \\
\hline
\end{tabular}

Table 1: Data of the reconstructed sources in the LR algorithm. FCX and FCY are the coordinates in imagepixels of the source in the FCFOV (FCFOV dimension are $99 \times 103$ pixels), CorrInt and LRInt are the reconstructed intensity in the case of CC and LR algorithm (counts $/ \mathrm{cm}^{2} / \mathrm{s}^{-1}$ ). In the last column we report a probable corrispondence with the CC OSA, the standard software of Integral analysis. The identification of D source is very uncertain.

PCFOV. If we look at the total FOV we see some edge effects at the corners of the reconstructed image. Instead, if we assume that sources could only be found in the FCFOV, we find a dirtier image. One advantage of the LR algorithm is that if we allow source construction throughout the FCFOV, no ghosts are created by the analysis and so no extra cleaning is required. 

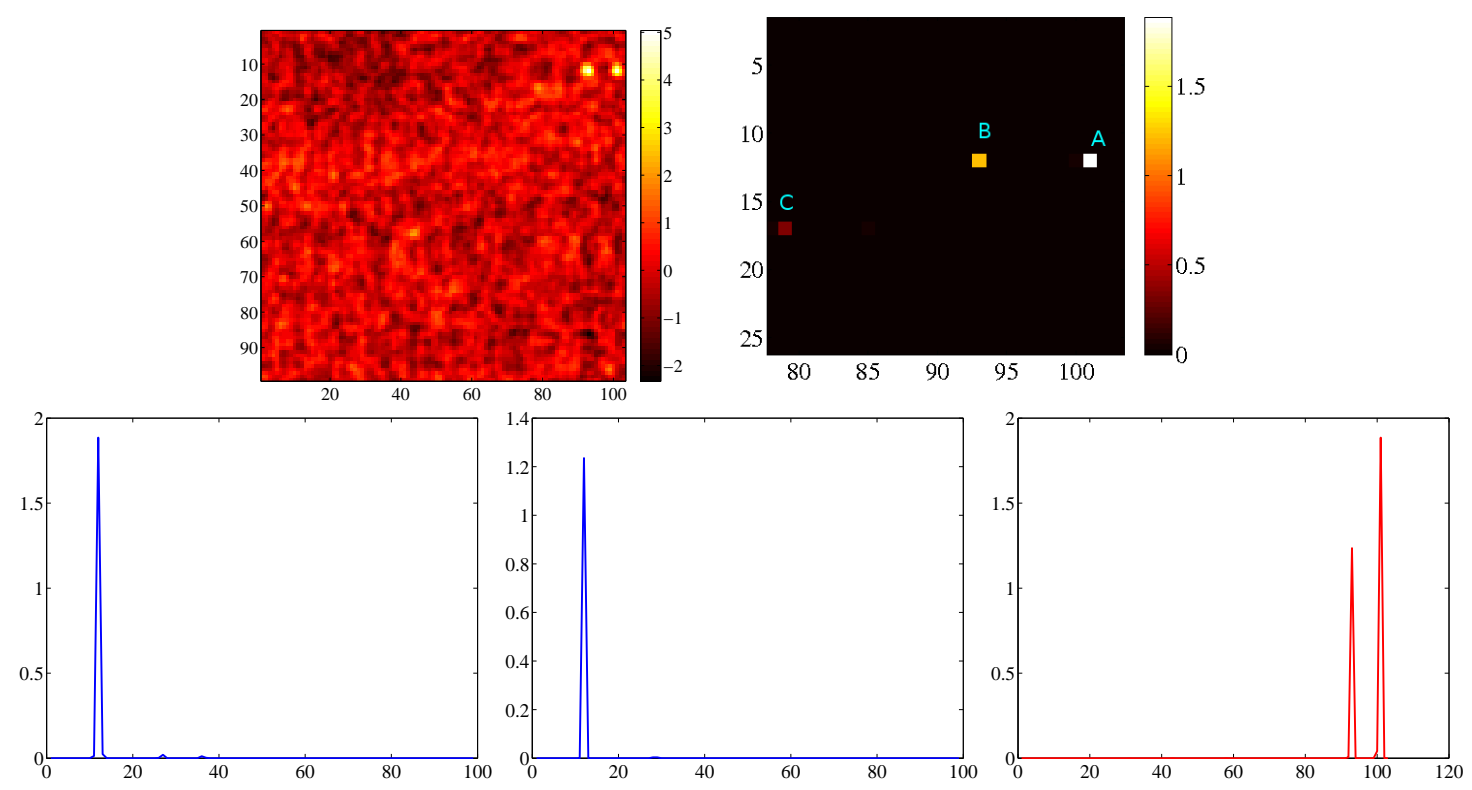

Figure 4: Galactic SCW analysis. (above) Images produced using the CC (left) and LR reconstruction algorithm (right); (below) Vertical (blue) and horizontal (red) cuts through the source positions for the LR reconstruction.

\section{Simulation of sources in the PCFOV.}

We simulate two sources: the first strong source is in the PCFOV and the second one is in the FCFOV and its intensity is one-third of that of the PCFOV source. It was supposed that there is the same probability to find sources in the FCFOV and in PCFOV. Fig.5 shows the reconstructions with CC and LR: while the CC shows a vertical features related to the source in the PCFOV, LR reconstructs both sources without any features.

Instead, if we suppose that there are sources only in the FCFOV, we find a reconstructed image
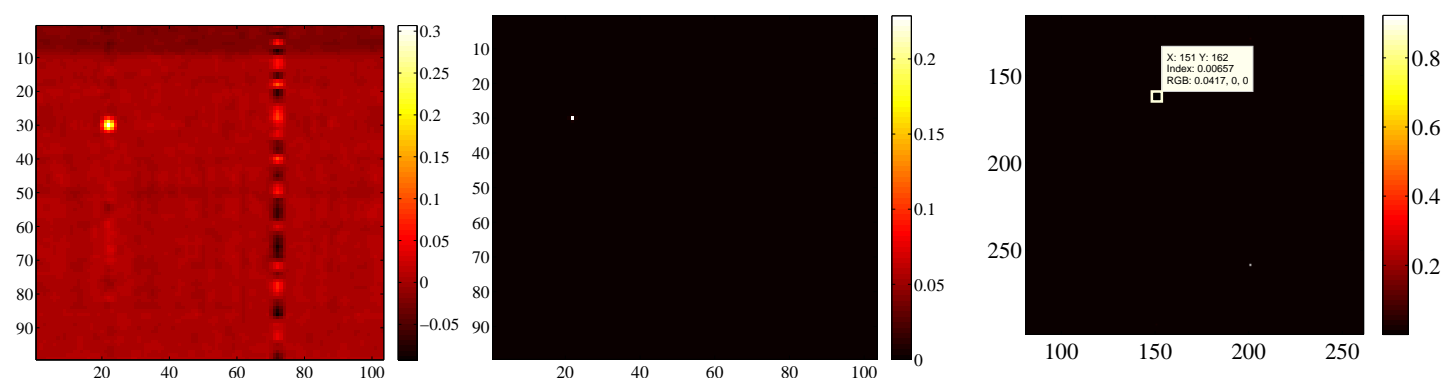

Figure 5: Simulation of one source in the PCFOV and one in the FCFOV: FCFOV reconstruction with CC and LR algorithm. Last picture is a zoom of the LR reconstruction of the total FOV.

similar to that found in the case of $\mathrm{CC}$, with the vertical feature related to the PCFOV source. 


\section{A first view: simulation of an extended source}

We simulate a 29-pixels source in the FCFOV. We have added a Poisson background distribution with constant mean value of 5000 counts/pxl and fixed the iteration number to the constant value of 200. In Fig. 6 we show some preliminary results.
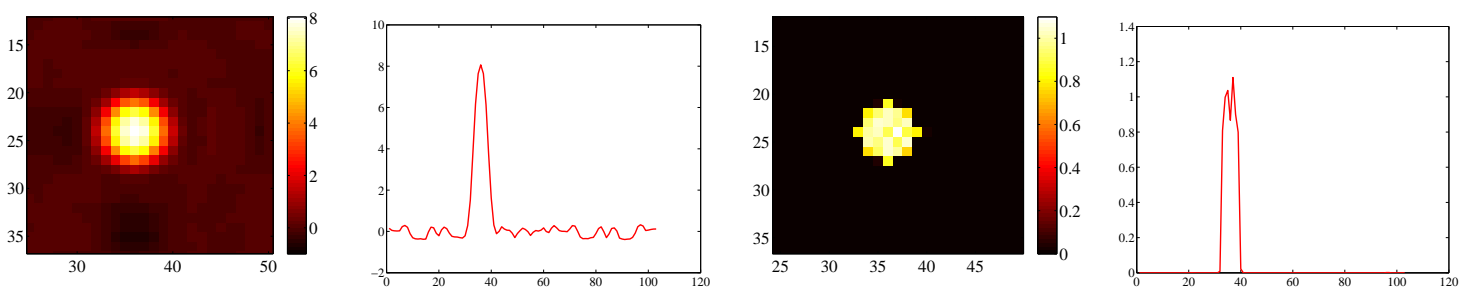

Figure 6: Extended source analysis: zoom of FCFOV reconstructions and line-profiles (vertical one) with standard CC software (left) and LR algorithm (right).

\section{Conclusion}

Even though this investigation is in the very early stages, we can see that the use of advanced decoding techniques such as the modified LR described here could be useful in extracting the maximum amount of information possible both from instruments without strong intrinsic angular resolution as well as those, such as coded aperture imagers which have already a good imaging capacity. In particular it is clear that the maximum angular resolution obtainable, at least in the case of strong sources, is not determined by the mask element dimensions but mainly by the detector pixel size. This investigation is continuing computing physical quantities as SNR, flux and errors. We will use both more complex simulations in conjunction with more crowded INTEGRAL/IBIS fields and the mosaicing of series of exposures.

\section{References}

[1] D.S.C. Biggs and M. Andrews, Acceleration of iterative image restoration algorithms, Applied Optics 368,1997

[2] E. Caroli, J.B. Stephen, G. di Cocco et al., Coded aperture imaging in X-and gamma-ray astronomy, Space Science Reviews, 45, 349-403, 1987

[3] Y. Chen, T.P. Li and M. Wu, Imaging by an Optimizing Method, Astronomical Data Analysis Software and Systems VI, ASP Conference Series, 25, 1997

[4] F. Lebrun, J.P. Leray, P. Lavocat et al, ISGRI: The INTEGRAL Soft Gamma-Ray Imager, A\&A 411, L141-L148, 2003 [arXiv:astro-ph/0310362v1]

[5] T.P. Li, Imaging in Harx X-ray Astronomy, 2002, [arXiv : astro.ph/0212022v1]

[6] L.B. Lucy, An Iterative Technique for the Rectification of Observed Distributions, Astronom.J. 79, 745-754, 1974

[7] W.H. Richardson, Baesian-Based Iterative Method of Image Restoration, J.Opt.Soc.Amer.A, 62, $55-59,1972$

[8] P. Ubertini, F. Lebrun et al., IBIS: the imager on-board INTEGRAL, A\&A, 411, L131, 2003 